\title{
Risk of Pneumonia is associated with Antipsychotic Drug Use among older patients with Parkinson's Disease: A Case-control Study
}

\author{
Kuang-Hua Huang ${ }^{*}$, Wei-Yin Kuo ${ }^{1 *}$, Yu-Hsiang Kuan ${ }^{2,3}$, Yu-Chia Chang, ${ }^{4,5}$, Tung-Han Tsai ${ }^{1}$, Chien-Ying Lee ${ }^{2,3 凶}$ \\ 1. Department of Health Services Administration, China Medical University, Taichung, Taiwan. \\ 2. Department of Pharmacology, Chung Shan Medical University, Taichung, Taiwan. \\ 3. Department of Pharmacy, Chung Shan Medical University Hospital, Taichung, Taiwan. \\ 4. Department of Healthcare Administration, Asia University, Taichung, Taiwan. \\ 5. Department of Long Term Care, National Quemoy University, Kinmen, Taiwan. \\ * These authors contributed equally to this work. \\ $\triangle$ Corresponding author: Chien-Ying Lee, PhD, Department of Pharmacology, Chung Shan Medical University, 110 Jian-Guo North Road, Section 1, Taichung \\ 40242, Taiwan, R.O.C. Tel: 886-4-24730022 \#11664. E-mail: cshd015@csmu.edu.tw.
}

(C) The author(s). This is an open access article distributed under the terms of the Creative Commons Attribution License (https://creativecommons.org/licenses/by/4.0/). See http://ivyspring.com/terms for full terms and conditions.

Received: 2021.05.27; Accepted: 2021.08.09; Published: 2021.08.26

\begin{abstract}
Objective: To investigate the risk of pneumonia associated with the use of antipsychotic drugs in older-adult patients with Parkinson's disease (PD) in Taiwan.

Methods: This case-control study was based on data from the longitudinal health insurance database in Taiwan. We analyzed the data of 51,158 older patients with PD for the period between 2001 and 2016. To reduce the potential confounding caused by unbalanced covariates in nonexperimental settings, we used propensity score matching to include older patients without pneumonia to serve as the control group.

Results: Compared with patients who had never taken antipsychotics, current (adjusted odds ratios [aOR] $=1.63,95 \%$ confidence interval $[\mathrm{Cl}]=1.51-1.75)$, recent $(\mathrm{aOR}=1.63,95 \% \mathrm{Cl}=1.52-1.74)$, and past $(\mathrm{aOR}=$ $1.89,95 \% \mathrm{Cl}=1.80-2.00)$ users of antipsychotics had a higher risk of incident pneumonia. Among typical and atypical antipsychotics, haloperidol and clozapine were associated with higher risks of incident pneumonia, respectively. By contrast, aripiprazole was not associated with a higher risk of pneumonia.

Conclusion: Older patients with PD receiving typical antipsychotics or atypical antipsychotics had a higher risk of pneumonia. Among these antipsychotics, clozapine had the highest risk of pneumonia. Clinicians should pay attention to the risk of pneumonia in older patients with PD who receive typical antipsychotics and atypical antipsychotics.
\end{abstract}

Key words: pneumonia, antipsychotics, Parkinson's disease, pharmacoepidemiology

\section{Introduction}

Parkinson's disease (PD) is the second most common age-related motor neurodegenerative disorder first described in the early 1800s by James Parkinson [1]. As PD progresses, the bulbar muscles are affected, leading to dysphagia. Dysphagia is a common symptom in patients with PD and may occur at any stage in the disease course. Most patients with PD develop oropharyngeal dysphagia in the early stage [2]. Oropharyngeal dysphagia is the sensation of difficulty or an abnormal delay in the movement of a food bolus from the oropharynx to the stomach. Those with PD often report difficulty swallowing, which is also associated with a high risk of aspiration pneumonia [3]. A 10-fold higher incidence of aspiration pneumonia was observed in patients with PD compared with patients without PD [4]. A meta-analysis revealed that the risk of oropharyngeal dysphagia in individuals with PD was approximately three times greater than that in healthy older adults [5]. Aspiration pneumonia is the leading cause of death in patients with PD, and it is estimated to account for $70 \%$ of the mortality in this group [6]. 
More than half of all patients with PD eventually develop PD psychosis, typically after 10 or more years of treatment [7]. The prevalence of various psychiatric disorders is high among those with PD. Antipsychotic drugs are often used for the treatment of behavioral and psychological symptoms in patients with PD [8]. Several observational studies have explored the relationship between the use of antipsychotic drugs and the risk of community-acquired pneumonia, mainly in older patients [9-11]. A systematic review and meta-analysis suggested that exposure to typical and atypical antipsychotic drugs is associated with a significantly increased risk of pneumonia in all age groups [12]. Only one study indicated that the risk of pneumonia was significantly higher for patients with PD who used unsuitable second-generation antipsychotic drugs compared with those taking the appropriate drugs, according to the American Geriatrics Society (AGS) 2015 Beers criteria in older patients [13].

However, few studies have examined the risk of pneumonia associated with the use of antipsychotic medications among patients with PD. Because antipsychotic agents are associated with an increased risk of pneumonia, we hypothesized that patients with PD have a higher risk of all-cause pneumonia. Understanding the risk of pneumonia in patients with PD is critical for clinicians. Therefore, we examined the risk of pneumonia associated with the use of antipsychotic drugs in older patients with PD and investigated the related risk factors for pneumonia by using nationwide data from Taiwan's longitudinal health insurance database (LHID).

\section{Methods}

\section{Database}

This study was a case-control study, in which secondary data analysis was conducted. Data were obtained from the LHID released by the Health and Welfare Data Science Center, Ministry of Health and Welfare Taiwan (HWDC, MOHW). The LHID comprises the information of two million beneficiaries randomly selected from the Taiwan National Health Insurance (NHI) program. The NHI program is a nationwide social insurance program that has covered up to $99 \%$ of citizens since 1995 . Hence, the database is nationally representative of Taiwan. Owing to the anonymity of the database, the requirement for informed consent was waived, and this study was approved as an ethical review by the Institutional Review Board of China Medical University Hospital, Taiwan (No. CMUH107-REC2-004).

\section{Study participants}

All study participants were older patients (aged $\geq 65$ years) with PD (International Classification of Diseases, Ninth Revision, Clinical Modification [ICD9-CM]: 332; International Classification of Diseases, Tenth Revision, Clinical Modification [ICD-10-CM]: G20) at any period from 2001 to 2016. Older patients who received a principal diagnosis of pneumonia (ICD-9-CM: 480-486, ICD-10-CM: J12-J18) comprised the case group. To reduce the potential confounding caused by unbalanced covariates in nonexperimental settings, we used 1:4 propensity score matching to include older patients without pneumonia to serve as the control group. The propensity score of the study was the probability of patients incident pneumonia, calculated by sex, age, income level, urbanization, and Charlson comorbidity index (CCI).

\section{Study design}

In this case-control study, we investigated the risk of pneumonia associated with incident antipsychotic use among older patients with PD. We defined antipsychotic use by the following Anatomic Therapeutic Chemical classification system codes: typical antipsychotics-haloperidol (N05AD01) and chlorpromazine (N05AA01)-and atypical antipsychotics-clozapine (N05AH02), olanzapine (N05AH03), quetiapine (N05AH04), aripiprazole (N05AX12), and risperidone (N05AX08). The observation period for assessing the antipsychotic use of each patient was the full year before the pneumonia diagnosis. The study calculated the duration of receiving antipsychotic drugs for each study subject. The definition of "current use" was the medication duration covered the date of incident pneumonia or ended at most 30 days before pneumonia; Antipsychotic use was categorized as "recent use" if the medication duration ended 31-90 days before pneumonia; If the medication duration ended more than 90 days was defined as "past use". Patients who had never been prescribed an antipsychotic before their pneumonia diagnosis served as the reference group.

\section{Statistical analysis}

We investigated the association between antipsychotic drugs and pneumonia through a conditional logistic regression. Each antipsychotic drug was seen as an independent variable. The study subjects may receive two or more kinds of antipsychotic drugs in the observation period. The conditional logistic regression analysis would estimate the odds ratio of each antipsychotic by adjusted all independent variables. The control variables were sex, age, income level, urbanization, CCI score, and comorbidities related to pneumonia. The following comorbidities were considered: diabetes mellitus (ICD-9-CM: 250; ICD-10-CM: E08- 
E13), hypertension (ICD-9-CM: 401-405; ICD-10-CM: I10-I13, I15), cerebrovascular disease (ICD-9-CM: 430-438; ICD-10-CM: I60-I69), arrhythmia (ICD-9CM: 427; ICD-10-CM: I47-I49), upper respiratory tract infection (ICD-9-CM: 465.9; ICD-10-CM: J00-06, J3039), heart failure (ICD-9-CM: 428.0; ICD-10-CM: I50), asthma (ICD-9-CM: 493, ICD-10-CM: J45), chronic obstructive pulmonary disease (COPD; ICD-9-CM: 490-492, 494-496; ICD-10-CM: J40-J44, J47), periodontitis (ICD-9-CM: 523; ICD-10-CM: K05.4), chronic kidney disease (ICD-9-CM: 585; ICD-10-CM: N18), liver disease (ICD-9-CM: 571; ICD-10-CM: K70K76), alcoholism (ICD-9-CM: 303, ICD-10-CM: F10.2), Alzheimer's disease (ICD-9-CM: 331.0, 290.1; ICD-10CM: G30, F00), rheumatoid arthritis (ICD-9-CM: 714; ICD-10-CM: M05-M06, M45), cancer (ICD-9-CM: 140239; ICD-10-CM: C00-C97), and epilepsy (ICD-9-CM: 345; ICD-10-CM: G40-G41). SAS version 9.4 (SAS Institute Inc., Cary, NC, USA) was used for statistical analysis, and statistical significance was indicated if $\mathrm{p}$ $<0.05$.

Table 1. Baseline characteristics of older patients with Parkinson's disease

\begin{tabular}{|c|c|c|c|c|c|}
\hline \multirow[t]{3}{*}{ Variables } & \multicolumn{4}{|c|}{ Pneumonia } & \multirow[t]{3}{*}{ p-value } \\
\hline & \multicolumn{2}{|c|}{ Without } & \multicolumn{2}{|l|}{ With } & \\
\hline & $\mathrm{N}$ & $\%$ & $\mathrm{~N}$ & $\%$ & \\
\hline Total & 40,948 & 100.00 & 10,237 & 100.00 & \\
\hline Gender & & & & & 0.078 \\
\hline Female & 21,750 & 53.12 & 5,338 & 52.14 & \\
\hline Male & 19,198 & 46.88 & 4,899 & 47.86 & \\
\hline Age (year) (mean $\pm S D)$ & \multicolumn{2}{|c|}{$77.03 \pm 5.85$} & \multicolumn{2}{|c|}{$77.10 \pm 5.84$} & 0.626 \\
\hline $65-70$ & 4,771 & 11.65 & 1,174 & 11.47 & \\
\hline $70-75$ & 8,396 & 20.50 & 2,109 & 20.60 & \\
\hline $75-80$ & 13,537 & 33.06 & 3,368 & 32.90 & \\
\hline $80-85$ & 11,921 & 29.11 & 3,037 & 29.67 & \\
\hline$\geq 85$ & 2,323 & 5.67 & 549 & 5.36 & \\
\hline Income level & & & & & 0.267 \\
\hline Low income $(\leq 21,000)$ & 21,784 & 53.20 & 5,476 & 53.49 & \\
\hline Middle income $(21,000-33,000)$ & 11,528 & 28.15 & 2,922 & 28.54 & \\
\hline High income $(\geq 33,000)$ & 7,636 & 18.65 & 1,839 & 17.96 & \\
\hline Urbanization & & & & & 0.622 \\
\hline Urban & 27,298 & 66.67 & 6,810 & 66.52 & \\
\hline Suburban & 9,042 & 22.08 & 2,299 & 22.46 & \\
\hline Rural & 4,608 & 11.25 & 1,128 & 11.02 & \\
\hline CCI score & & & & & 0.827 \\
\hline 0 & 6,977 & 17.04 & 1,714 & 16.74 & \\
\hline 1 & 9,701 & 23.69 & 2,457 & 24.00 & \\
\hline 2 & 9,762 & 23.84 & 2,456 & 23.99 & \\
\hline$\geq 3$ & 14,508 & 35.43 & 3,610 & 35.26 & \\
\hline Diabetes mellitus & & & & & $<0.001$ \\
\hline No & 25,104 & 61.31 & 6,696 & 65.41 & \\
\hline Yes & 15,844 & 38.69 & 3,541 & 34.59 & \\
\hline Hypertension & & & & & $<0.001$ \\
\hline No & 11,091 & 27.09 & 3,207 & 31.33 & \\
\hline Yes & 29,857 & 72.91 & 7,030 & 68.67 & \\
\hline Cerebrovascular disease & & & & & $<0.001$ \\
\hline No & 19,668 & 48.03 & 4,112 & 40.17 & \\
\hline Yes & 21,280 & 51.97 & 6,125 & 59.83 & \\
\hline Arrhythmia & & & & & 0.134 \\
\hline No & 33,605 & 82.07 & 8,336 & 81.43 & \\
\hline Yes & 7,343 & 17.93 & 1,901 & 18.57 & \\
\hline Upper respiratory tract infection & & & & & 0.944 \\
\hline No & 19,948 & 48.72 & 4,983 & 48.68 & \\
\hline Yes & 21,000 & 51.28 & 5,254 & 51.32 & \\
\hline
\end{tabular}

\begin{tabular}{|c|c|c|c|c|c|}
\hline \multirow[t]{3}{*}{ Variables } & \multicolumn{4}{|c|}{ Pneumonia } & \multirow[t]{3}{*}{ p-value } \\
\hline & \multicolumn{2}{|c|}{ Without } & \multicolumn{2}{|l|}{ With } & \\
\hline & $\mathrm{N}$ & $\%$ & $\mathrm{~N}$ & $\%$ & \\
\hline Heart failure & & & & & $<0.001$ \\
\hline No & 34,740 & 84.84 & 8,398 & 82.04 & \\
\hline Yes & 6,208 & 15.16 & 1,839 & 17.96 & \\
\hline Asthma & & & & & $<0.001$ \\
\hline No & 35,733 & 87.26 & 7,989 & 78.04 & \\
\hline Yes & 5,215 & 12.74 & 2,248 & 21.96 & \\
\hline COPD & & & & & $<0.001$ \\
\hline No & 28,002 & 68.38 & 4,301 & 42.01 & \\
\hline Yes & 12,946 & 31.62 & 5,936 & 57.99 & \\
\hline Periodontitis & & & & & 0.155 \\
\hline No & 40,017 & 97.73 & 9,980 & 97.49 & \\
\hline Yes & 931 & 2.27 & 257 & 2.51 & \\
\hline Chronic kidney disease & & & & & $<0.001$ \\
\hline No & 39,657 & 96.85 & 9,980 & 97.49 & \\
\hline Yes & 1,291 & 3.15 & 257 & 2.51 & \\
\hline Chronic liver disease & & & & & $<0.001$ \\
\hline No & 35,193 & 85.95 & 9,141 & 89.29 & \\
\hline Yes & 5,755 & 14.05 & 1,096 & 10.71 & \\
\hline Alcoholism & & & & & $<0.001$ \\
\hline No & 40,901 & 99.89 & 10,202 & 99.66 & \\
\hline Yes & 47 & 0.11 & 35 & 0.34 & \\
\hline Alzheimer disease & & & & & $<0.001$ \\
\hline No & 36,129 & 88.23 & 8,357 & 81.64 & \\
\hline Yes & 4,819 & 11.77 & 1,880 & 18.36 & \\
\hline Rheumatoid arthritis & & & & & 0.078 \\
\hline No & 39,789 & 97.17 & 9,980 & 97.49 & \\
\hline Yes & 1,159 & 2.83 & 257 & 2.51 & \\
\hline Cancer & & & & & $<0.001$ \\
\hline No & 33,008 & 80.61 & 8,402 & 82.07 & \\
\hline Yes & 7,940 & 19.39 & 1,835 & 17.93 & \\
\hline Epilepsy & & & & & $<0.001$ \\
\hline No & 39,161 & 95.64 & 9,039 & 88.30 & \\
\hline Yes & 1,787 & 4.36 & 1,198 & 11.70 & \\
\hline Schizophrenia & & & & & $<0.001$ \\
\hline No & 40,263 & 98.33 & 9,948 & 97.18 & \\
\hline Yes & 685 & 1.67 & 289 & 2.82 & \\
\hline Bipolar disorder & & & & & $<0.001$ \\
\hline No & 40,009 & 97.71 & 9,869 & 96.41 & \\
\hline Yes & 939 & 2.29 & 368 & 3.59 & \\
\hline Major depressive disorder & & & & & 0.082 \\
\hline No & 38,291 & 93.51 & 9,524 & 93.04 & \\
\hline Yes & 2,657 & 6.49 & 713 & 6.96 & \\
\hline Anxiety & & & & & $<0.001$ \\
\hline No & 39,431 & 96.30 & 10,047 & 98.14 & \\
\hline Yes & 1,517 & 3.70 & 190 & 1.86 & \\
\hline
\end{tabular}

Results

\section{Baseline characteristics of the sample}

Table 1 presents the baseline characteristics of the sample. After matching was conducted, the data of 51,158 older patients with PD were included for analysis, including 10,237 and 40,948 patients with and without incident pneumonia, respectively. The mean age of the patients with pneumonia was $77.10 \pm$ 5.84 years. As expected, the distributions of sex, age, income level, urbanization, and CCI did not significantly differ between the case and control groups after matching. In the case group, $34.59 \%$ of patients had diabetes mellitus, $68.67 \%$ had hypertension, $59.83 \%$ had cerebrovascular disease, $18.57 \%$ had arrhythmia, $51.32 \%$ had upper respiratory tract infection, $17.96 \%$ had heart failure, $21.96 \%$ had asthma, $57.99 \%$ had COPD, $2.51 \%$ had periodontitis, 
$2.51 \%$ had chronic kidney disease, $10.71 \%$ had chronic liver disease, $18.36 \%$ had Alzheimer disease, 2.51\% had rheumatoid arthritis, $17.93 \%$ had cancer, $11.70 \%$ had epilepsy, and $6.96 \%$ had major depressive disorder.

\section{Incidence of pneumonia with antipsychotic use}

Table 2 lists the incidence rate of pneumonia with antipsychotic use. The incidence rate of pneumonia was $19.06 \%$ in patients who had never received any antipsychotics, $30.49 \%$ in current users, $29.88 \%$ in recent users, and $31.39 \%$ in past users ( $p<$ 0.001). As for typical antipsychotic use, incident pneumonia was diagnosed in $19.84 \%$ of patients who had never taken any antipsychotics, in $35.23 \%$ of current users, in $31.17 \%$ of recent users, and in $28.69 \%$ of past users ( $p$ 0.001). As for atypical antipsychotics use, incident pneumonia was diagnosed in $19.22 \%$ of patients who had never taken any antipsychotics, in $29.85 \%$ of current users, in $29.71 \%$ of recent users, and in $32.28 \%$ of past users $(\mathrm{p}<0.001)$.

The distribution of incident pneumonia among the usage groups differed significantly for individual typical antipsychotics $(\mathrm{p}<0.001)$. Incident pneumonia also differed significantly between each atypical antipsychotic, except for aripiprazole.

\section{Association of incident pneumonia and antipsychotic use}

Table 3 reveals the adjusted odds ratios (aORs) for antipsychotics after sex, age, income level, urbanization, and related comorbidities were controlled for. Compared with patients who had never taken antipsychotics, those currently taking antipsychotics had a higher risk of incident pneumonia $(\mathrm{aOR}=1.63,95 \%$ confidence interval $[\mathrm{CI}]$ $=1.51-1.75)$, recent users $(\mathrm{aOR}=1.63,95 \% \mathrm{CI}=1.52-$ 1.74 ), and past users (aOR $=1.89,95 \% \mathrm{CI}=1.80-2.00)$. As for typical antipsychotics, compared with patients who had never taken typical antipsychotics, incident pneumonia risk was higher in current users $(\mathrm{aOR}=$ $1.76,95 \% \mathrm{CI}=1.46-2.13)$, recent users $(\mathrm{aOR}=1.46$, $95 \% \mathrm{CI}=1.23-1.73)$, and past users $(\mathrm{aOR}=1.10,95 \%$ $\mathrm{CI}=1.00-1.21)$. As for atypical antipsychotics, compared with patients who had never taken atypical antipsychotics, incident pneumonia risk was higher in current users $(\mathrm{aOR}=1.57,95 \% \mathrm{CI}=1.45-1.70)$, recent users $(\mathrm{aOR}=1.61,95 \% \mathrm{CI}=1.50-1.73)$, and past users $(\mathrm{aOR}=1.94,95 \% \mathrm{CI}=1.83-2.05)$. Among typical antipsychotics, haloperidol had a higher risk of incident pneumonia for both current users $(\mathrm{aOR}=$ $1.72,95 \% \mathrm{CI}=1.41-2.11)$ and recent users $(\mathrm{aOR}=1.41$, $95 \% \mathrm{CI}=1.17-1.69$ ). For chlorpromazine, only current users had a higher risk $(\mathrm{aOR}=1.84,95 \% \mathrm{CI}=$ 1.13-2.98).
Table 2. Incidence of pneumonia in older patients with Parkinson's disease

\begin{tabular}{|c|c|c|c|c|c|}
\hline \multirow[t]{3}{*}{ Variables } & \multicolumn{4}{|c|}{ Pneumonia } & \multirow[t]{3}{*}{ p-value } \\
\hline & \multicolumn{2}{|c|}{ Without } & \multicolumn{2}{|l|}{ With } & \\
\hline & $\bar{N}$ & $\%$ & $\mathrm{~N}$ & $\%$ & \\
\hline \multicolumn{6}{|c|}{ Any one of Antipsychotic } \\
\hline No & 38,030 & 80.94 & 8,957 & 19.06 & \\
\hline Current users & 2,918 & 69.51 & 1,280 & 30.49 & $<0.001$ \\
\hline Recent users & 3,813 & 70.12 & 1,625 & 29.88 & $<0.001$ \\
\hline Past users & 6,959 & 68.61 & 3,184 & 31.39 & $<0.001$ \\
\hline \multicolumn{6}{|c|}{ Typical antipsychotics } \\
\hline No & 40,595 & 80.16 & 10,045 & 19.84 & \\
\hline Current users & 353 & 64.77 & 192 & 35.23 & $<0.001$ \\
\hline Recent users & 488 & 68.83 & 221 & 31.17 & $<0.001$ \\
\hline Past users & 1,904 & 71.31 & 766 & 28.69 & $<0.001$ \\
\hline \multicolumn{6}{|l|}{ Haloperidol } \\
\hline No & 40,649 & 80.14 & 10,074 & 19.86 & \\
\hline Current users & 299 & 64.72 & 163 & 35.28 & $<0.001$ \\
\hline Recent users & 429 & 69.08 & 192 & 30.92 & $<0.001$ \\
\hline Past users & 1,658 & 70.67 & 688 & 29.33 & $<0.001$ \\
\hline \multicolumn{6}{|c|}{ Chlorpromazine } \\
\hline No & 40,891 & 80.02 & 10,208 & 19.98 & \\
\hline Current users & 57 & 66.28 & 29 & 33.72 & 0.002 \\
\hline Recent users & 66 & 68.75 & 30 & 31.25 & 0.006 \\
\hline Past users & 299 & 70.85 & 123 & 29.15 & $<0.001$ \\
\hline \multicolumn{6}{|c|}{ Atypical antipsychotics } \\
\hline No & 38,328 & 80.78 & 9,122 & 19.22 & \\
\hline Current users & 2,620 & 70.15 & 1,115 & 29.85 & $<0.001$ \\
\hline Recent users & 3,407 & 70.29 & 1,440 & 29.71 & $<0.001$ \\
\hline Past users & 5,917 & 67.72 & 2,821 & 32.28 & $<0.001$ \\
\hline \multicolumn{6}{|l|}{ Clozapine } \\
\hline No & 40,851 & 80.04 & 10,185 & 19.96 & \\
\hline Current users & 97 & 65.10 & 52 & 34.90 & $<0.001$ \\
\hline Recent users & 122 & 58.37 & 87 & 41.63 & $<0.001$ \\
\hline Past users & 245 & 58.06 & 177 & 41.94 & $<0.001$ \\
\hline \multicolumn{6}{|l|}{ Olanzapine } \\
\hline No & 40,771 & 80.05 & 10,160 & 19.95 & \\
\hline Current users & 177 & 69.69 & 77 & 30.31 & $<0.001$ \\
\hline Recent users & 236 & 75.88 & 75 & 24.12 & 0.069 \\
\hline Past users & 611 & 66.41 & 309 & 33.59 & $<0.001$ \\
\hline \multicolumn{6}{|l|}{ Quetiapine } \\
\hline No & 39,128 & 80.45 & 9,509 & 19.55 & \\
\hline Current users & 1,820 & 71.43 & 728 & 28.57 & $<0.001$ \\
\hline Recent users & 2,391 & 70.89 & 982 & 29.11 & $<0.001$ \\
\hline Past users & 4,227 & 67.48 & 2,037 & 32.52 & $<0.001$ \\
\hline \multicolumn{6}{|l|}{ Aripiprazole } \\
\hline No & 40,878 & 80.01 & 10,212 & 19.99 & \\
\hline Current users & 70 & 73.68 & 25 & 26.32 & 0.124 \\
\hline Recent users & 87 & 75.00 & 29 & 25.00 & 0.178 \\
\hline Past users & 255 & 75.44 & 83 & 24.56 & 0.036 \\
\hline \multicolumn{6}{|l|}{ Risperidone } \\
\hline No & 40,425 & 80.23 & 9,960 & 19.77 & \\
\hline Current users & 523 & 65.38 & 277 & 34.63 & $<0.001$ \\
\hline Recent users & 708 & 67.11 & 347 & 32.89 & $<0.001$ \\
\hline Past users & 1,990 & 65.57 & 1,045 & 34.43 & $<0.001$ \\
\hline
\end{tabular}

Among atypical antipsychotics, clozapine had a higher risk of incident pneumonia regardless of whether its use was current $(\mathrm{aOR}=1.77,95 \% \mathrm{CI}=$ 1.24-2.51), recent $(\mathrm{aOR}=2.60,95 \% \mathrm{CI}=1.95-3.46)$, or in the past $(\mathrm{aOR}=1.81,95 \% \mathrm{CI}=1.46-2.23)$. Patients with current $(\mathrm{aOR}=1.60,95 \% \mathrm{CI}=1.21-2.51)$ or past $(\mathrm{aOR}=1.20,95 \% \mathrm{CI}=1.03-1.40)$ olanzapine use had a higher risk of incident pneumonia, compared with those who had never taken atypical antipsychotics. Finally, compared with those who had never taken atypical antipsychotics, both quetiapine users (current: $\mathrm{aOR}=1.44,95 \% \mathrm{CI}=1.31-1.58$; recent user: $\mathrm{aOR}=1.53,95 \% \mathrm{CI}=1.41-1.66$; past users: $\mathrm{aOR}=1.71$, 
95\% CI $=1.60-1.82$ ), and risperidone users (current user: $\mathrm{aOR}=1.81,95 \% \mathrm{CI}=1.55-2.11$; recent user: $\mathrm{aOR}$ $=1.62,95 \% \mathrm{CI}=1.41-1.86$; past users: $\mathrm{aOR}=1.52,95 \%$ $\mathrm{CI}=1.39-1.66)$ had $\mathrm{a}$ higher risk of incident pneumonia.

Table 3. Association between antipsychotic drugs and pneumonia

\begin{tabular}{|c|c|c|c|c|c|c|}
\hline \multirow[t]{2}{*}{ Variables } & \multicolumn{2}{|c|}{ Model 1 a } & \multicolumn{2}{|c|}{ Model 2 b } & \multicolumn{2}{|c|}{ Model 3c } \\
\hline & OR & $95 \% \mathrm{CI}$ & OR & $95 \% \mathrm{CI}$ & OR & $95 \% \mathrm{CI}$ \\
\hline \multicolumn{7}{|l|}{ Any one of Antipsychotic } \\
\hline No (ref.) & 1 & & & & & \\
\hline Current users & 1.63 & $1.51-1.75$ & - & - & - & - \\
\hline Recent users & 1.63 & $1.52-1.74$ & - & - & - & - \\
\hline Past users & 1.89 & $1.80-2.00$ & - & - & - & - \\
\hline \multicolumn{7}{|l|}{ Typical antipsychotics } \\
\hline No (ref.) & & & 1 & & & \\
\hline Current users & - & - & 1.76 & $1.46-2.13$ & - & - \\
\hline Recent users & - & - & 1.46 & $1.23-1.73$ & - & - \\
\hline Past users & - & - & 1.10 & $1.00-1.21$ & - & - \\
\hline \multicolumn{7}{|l|}{ Haloperidol } \\
\hline No (ref.) & & & & & 1 & \\
\hline Current users & - & - & - & - & 1.72 & $1.41-2.11$ \\
\hline Recent users & - & - & - & - & 1.41 & $1.17-1.69$ \\
\hline Past users & - & - & - & - & 1.08 & $0.97-1.19$ \\
\hline \multicolumn{7}{|l|}{ Chlorpromazine } \\
\hline No (ref.) & & & & & 1 & \\
\hline Current users & - & - & - & - & 1.84 & $1.13-2.98$ \\
\hline Recent users & - & - & - & - & 1.44 & $0.90-2.30$ \\
\hline Past users & - & - & - & - & 1.13 & $0.90-1.43$ \\
\hline \multicolumn{7}{|l|}{ Atypical antipsychotics } \\
\hline No (ref.) & & & 1 & & & \\
\hline Current users & - & - & 1.57 & $1.45-1.70$ & - & - \\
\hline Recent users & - & - & 1.61 & $1.50-1.73$ & - & - \\
\hline Past users & - & - & 1.94 & $1.83-2.05$ & - & - \\
\hline \multicolumn{7}{|l|}{ Clozapine } \\
\hline No (ref.) & & & & & 1 & \\
\hline Current users & - & - & - & - & 1.77 & $1.24-2.51$ \\
\hline Recent users & - & - & - & - & 2.60 & $1.95-3.46$ \\
\hline Past users & - & - & - & - & 1.81 & $1.46-2.23$ \\
\hline \multicolumn{7}{|l|}{ Olanzapine } \\
\hline No (ref.) & & & & & 1 & \\
\hline Current users & - & - & - & - & 1.60 & $1.21-2.13$ \\
\hline Recent users & - & - & - & - & 1.15 & $0.87-1.51$ \\
\hline Past users & - & - & - & - & 1.20 & $1.03-1.40$ \\
\hline \multicolumn{7}{|l|}{ Quetiapine } \\
\hline No (ref.) & & & & & 1 & \\
\hline Current users & - & - & - & - & 1.44 & $1.31-1.58$ \\
\hline Recent users & - & - & - & - & 1.53 & $1.41-1.66$ \\
\hline Past users & - & - & - & - & 1.71 & $1.60-1.82$ \\
\hline \multicolumn{7}{|l|}{ Aripiprazole } \\
\hline No (ref.) & & & & & 1 & \\
\hline Current users & - & - & - & - & 1.56 & $0.97-2.51$ \\
\hline Recent users & - & - & - & - & 1.46 & $0.94-2.26$ \\
\hline Past users & - & - & - & - & 0.98 & $0.75-1.27$ \\
\hline \multicolumn{7}{|l|}{ Risperidone } \\
\hline No (ref.) & & & & & 1 & \\
\hline Current users & - & - & - & - & 1.81 & $1.55-2.11$ \\
\hline Recent users & - & - & - & - & 1.62 & $1.41-1.86$ \\
\hline Past users & - & - & - & - & 1.52 & $1.39-1.66$ \\
\hline Comorbidities (Yes vs No) & & & & & & \\
\hline Diabetes mellitus & 0.88 & $0.84-0.92$ & 0.88 & $0.84-0.92$ & 0.88 & $0.84-0.92$ \\
\hline Hypertension & 0.74 & $0.70-0.78$ & 0.74 & $0.70-0.78$ & 0.74 & $0.70-0.78$ \\
\hline Cerebrovascular disease & 1.19 & $1.13-1.25$ & 1.19 & $1.13-1.25$ & 1.19 & $1.13-1.25$ \\
\hline Arrhythmia & 0.94 & $0.89-1.00$ & 0.94 & $0.89-1.00$ & 0.95 & $0.89-1.00$ \\
\hline Upper respiratory tract infection & 0.88 & $0.84-0.92$ & 0.88 & $0.84-0.92$ & 0.88 & $0.84-0.92$ \\
\hline Congestive heart failure & 1.08 & $1.01-1.15$ & 1.08 & 1.01-1.15 & 1.08 & $1.01-1.15$ \\
\hline Asthma & 1.37 & $1.29-1.46$ & 1.37 & $1.29-1.46$ & 1.37 & $1.29-1.46$ \\
\hline COPD & 2.73 & $2.60-2.86$ & 2.73 & $2.60-2.86$ & 2.73 & $2.60-2.86$ \\
\hline Periodontitis & 1.02 & $0.89-1.19$ & 1.02 & 0.89-1.19 & 1.02 & $0.88-1.18$ \\
\hline Chronic kidney disease & 0.97 & $0.84-1.11$ & 0.97 & $0.84-1.11$ & 0.97 & $0.84-1.11$ \\
\hline Chronic liver disease & 0.70 & $0.65-0.75$ & 0.70 & $0.65-0.75$ & 0.69 & $0.65-0.75$ \\
\hline
\end{tabular}

\begin{tabular}{|c|c|c|c|c|c|c|}
\hline \multirow[t]{2}{*}{ Variables } & \multicolumn{2}{|c|}{ Model 1 a } & \multicolumn{2}{|c|}{ Model 2 b } & \multicolumn{2}{|c|}{ Model 3c } \\
\hline & $\overline{\mathrm{OR}}$ & $95 \% \mathrm{CI}$ & OR & $95 \% \mathrm{CI}$ & OR & $95 \% \mathrm{CI}$ \\
\hline Alcoholism & 1.91 & $1.22-3.01$ & 1.89 & $1.20-2.98$ & 1.89 & $1.19-2.99$ \\
\hline Alzheimer disease & 1.35 & $1.27-1.44$ & 1.36 & $1.27-1.44$ & 1.36 & $1.28-1.44$ \\
\hline Rheumatoid arthritis & 0.85 & $0.74-0.98$ & 0.85 & $0.74-0.98$ & 0.85 & $0.74-0.98$ \\
\hline Cancer & 0.87 & $0.82-0.92$ & 0.87 & $0.81-0.92$ & 0.87 & $0.82-0.92$ \\
\hline Epilepsy & 2.14 & $1.98-2.33$ & 2.14 & $1.98-2.33$ & 2.15 & $1.98-2.33$ \\
\hline Schizophrenia & 1.33 & $1.15-1.54$ & 1.32 & $1.14-1.54$ & 1.30 & $1.12-1.51$ \\
\hline Bipolar disorder & 1.41 & $1.23-1.61$ & 1.42 & $1.24-1.62$ & 1.41 & $1.24-1.62$ \\
\hline Major depressive disorder & 0.94 & $0.85-1.03$ & 0.94 & $0.85-1.03$ & 0.94 & $0.86-1.03$ \\
\hline Anxiety & 0.59 & $0.50-0.69$ & 0.59 & $0.50-0.69$ & 0.59 & $0.50-0.69$ \\
\hline
\end{tabular}

Patients with diabetes mellitus, hypertension, upper respiratory tract infection, chronic liver disease, rheumatoid arthritis, cancer, and anxiety had a lower risk of incident pneumonia, whereas patients with cerebrovascular disease, cerebrovascular disease, asthma, COPD, alcoholism, Alzheimer disease, epilepsy, schizophrenia, and bipolar disorder had a higher risk of incident pneumonia.

\section{Discussion}

Oropharyngeal dysphagia is a frequent symptom in neurological disease. In neurodegenerative disorders such as PD and related disorders, as the disease progresses, patients have an increased risk of rapidly developing dysphagia [5]. Patients with PD are at greater risk of aspiration pneumonia than are individuals in the general population [14]. Because no studies have examined whether those with PD who use antipsychotic drugs also have a higher risk of pneumonia, we investigated the risk of pneumonia associated with the use of antipsychotic drugs among older patients with PD.

Antipsychotics have varying degrees of anticholinergic effects and could lead to aspiration pneumonia because of dry mouth and impaired oropharyngeal bolus transport [15]. Anticholinergic drug use is a risk factor for pneumonia in older patients. A Taiwanese study indicated that older patients receiving anticholinergic drugs have an increased risk of incident pneumonia [16]. One established mechanism for pneumonia is dry mouth, which is frequently caused by the anticholinergic side effects of medications; a dry mouth may lead to oropharyngeal swallowing impairment, which may result in aspiration pneumonia and even lead to death [17]. Anticholinergic burden scores for drugs in Germany indicated that antipsychotics such as aripiprazole and risperidone have weak anticholinergic effects (score $=$ 1); haloperidol, olanzapine, and quetiapine have moderate anticholinergic effects (score $=2$ ); and clozapine has strong anticholinergic effects (score $=3$ ) [18]. Another scale for anticholinergic activity drugs used in Brazil indicated that antipsychotics such as 
aripiprazole, haloperidol, and risperidone have weak anticholinergic effects (score $=1$ ); olanzapine and quetiapine have moderate anticholinergic effects (score $=2$ ); and clozapine and chlorpromazine have strong anticholinergic effects (score $=3$ ) [19]. Based on these two scales, we included seven drugs, namely haloperidol, chlorpromazine, olanzapine, quetiapine, risperidone, clozapine, and aripiprazole, to investigate the relationship between antipsychotic drugs and pneumonia risk among older patients with PD. Our results indicate that older patients with PD who receive antipsychotics had a higher risk of pneumonia. Compared with patients who had never taken antipsychotics, current users, $(\mathrm{aOR}=1.63,95 \%$ $\mathrm{CI}=1.51-1.75)$, recent users $(\mathrm{aOR}=1.63,95 \% \mathrm{CI}=$ $1.52-1.74)$, and past users $(\mathrm{aOR}=1.89,95 \% \mathrm{CI}=$ 1.80-2.00) had a higher risk of incident pneumonia.

Persistent psychotic symptoms may develop in up to $40 \%-60 \%$ of patients with PD, but estimates vary widely [7, 20, 21]. Evidence suggests that people with psychiatric disorders are at an increased risk of common infectious diseases [22]. For the treatment of patients with PD, the use of atypical antipsychotics (e.g., clozapine, pimavanserin, or quetiapine) should be prioritized; typical antipsychotics (e.g., haloperidol) are not recommended [23]. A metaanalysis suggested that the risk of pneumonia was significantly increased by exposure to typical antipsychotic drugs $(\mathrm{OR}=1.68,95 \% \mathrm{CI}=1.39-2.04)$ and atypical antipsychotic drugs $(\mathrm{OR}=1.98,95 \% \mathrm{CI}=$ 1.67-2.35) in all age groups [12]. Our study also revealed that older patients with $\mathrm{PD}$ who receive typical antipsychotics and atypical antipsychotics had a higher risk of incident pneumonia.

Our results indicated that current, recent, or past use of clozapine leads to the highest risk of pneumonia of all the studied antipsychotic medications; this result may be attributed to clozapine's strong anticholinergic effect $[18,19]$. We also found that those taking aripiprazole, an atypical antipsychotic, did not have an elevated risk of pneumonia; this finding may be attributed to aripiprazole's weak anticholinergic effect $[18,19]$. The scale used in Brazil indicated that chlorpromazine has a strong anticholinergic effect [20], whereas data from Germany indicated that chlorpromazine has no anticholinergic effect [19]. Thus, further investigation is required to clarify the causal relationship between chlorpromazine and pneumonia. Antipsychotics have immunoregulatory effects and anti-inflammatory effects, and clozapine may have inflammatory effects [24]. Clozapine exposure may be associated with an increased risk of pneumonia when used individually and with an even higher risk when treatment is combined with other antipsychotics [25]. Some atypical antipsychotics, such as clozapine, quetiapine, or risperidone, can modulate the cytokine network $[26,27]$, and clozapine directly influences the plasma levels of several cytokines that resemble an inflammatory reaction [24]; clozapine might also enhance susceptibility to infections during treatment [28].

The main strength of our study is that it is the first to investigate the relationship between antipsychotic use and pneumonia risk in older patients with PD by dividing the follow-up period into current use, recent use, and past use. Compared with patients who had never taken typical antipsychotics, current $(\mathrm{aOR}=1.76,95 \% \mathrm{CI}=$ 1.46-2.13), recent ( $\mathrm{aOR}=1.46,95 \% \mathrm{CI}=1.23-1.73)$, and past $(\mathrm{aOR}=1.10,95 \% \mathrm{CI}=1.00-1.21)$ users of typical antipsychotics had a higher risk of incident pneumonia. Furthermore, compared with patients who had never taken atypical antipsychotics, current $(\mathrm{aOR}=1.57,95 \% \mathrm{CI}=1.45-1.70)$, recent $(\mathrm{aOR}=1.61$, $95 \% \mathrm{CI}=1.50-1.73)$, and past $(\mathrm{aOR}=1.94,95 \% \mathrm{CI}=$ 1.83-2.05) users of atypical antipsychotics had a higher risk of incident pneumonia. Our study revealed that older patients with PD with current, recent, or past use of haloperidol, olanzapine, quetiapine, risperidone, or clozapine had a higher risk of pneumonia, whereas only those currently using chlorpromazine had a higher risk of pneumonia; by contrast, those taking aripiprazole did not have an increased risk of pneumonia.

To the best of our knowledge, ours is the first study to identify the risk factors for pneumonia in those with PD who receive antipsychotic drugs. A univariate Cox proportional hazards analysis indicated that patients with PD with comorbid cerebrovascular disease, congestive heart failure, asthma, COPD, alcoholism, Alzheimer's disease, or epilepsy had a lower risk of incident pneumonia. Our study also revealed that in patients with PD, combined psychiatric disorder (schizophrenia and bipolar disorder) is an independent predictive factor for the development of pneumonia in patients with PD. The risk of pneumococcal disease in each psychiatric group was significantly higher than that for the general population. Patients with psychiatric disorders (e.g., schizophrenia, bipolar disorder, depression, and anxiety) have an increased risk of pneumonia [29]. Patients with schizophrenia have an increased risk of pneumonia [30], and all-cause mortality is higher in this group than in the general population [31]. This finding is supported by earlier investigations on the in vitro production of cytokines in people with these disorders [24]. Another study indicated that cerebrovascular disease, congestive heart failure, COPD, and epilepsy are associated with 
a higher risk of pneumonia in those with PD [32].

Typical antipsychotics act predominantly through dopamine $\mathrm{D}_{2}$ receptor antagonism, which also exacerbates parkinsonian motor deficits [33]. According to the AGS 2015 Beers criteria, atypical antipsychotics (except for aripiprazole, clozapine, and quetiapine) are unsuitable for patients with PD patients because of the risk of worsening parkinsonian symptoms [34]. According to the 2019 Beers criteria, for patients with PD, all antipsychotics have the potential to aggravate parkinsonian symptoms; however, pimavanserin and clozapine appear to be less likely to precipitate worsening of PD. Quetiapine has only been studied in low-quality clinical trials with efficacy reported in five trials and efficacy similar to that of clozapine reported in two others [35]. Those with PD treated with quetiapine exhibit increased mortality, with a higher risk of death compared with those not using an antipsychotic; similar results were also observed for olanzapine and risperidone but not for clozapine [36]. These data indicate that clozapine may be a suitable medication for individuals with PD who experience psychotic symptoms.

In a study conducted according to the 2015 Beers criteria, Cox regression analyses revealed an increased risk of pneumonia in nursing home residents with $\mathrm{PD}$ who were taking inappropriate antipsychotic agents compared with those taking appropriate agents [13]. Older people with PD in long-term care who have therapy-related psychosis and use inappropriate antipsychotic medications may experience a deterioration in parkinsonian symptoms [37]. Inappropriate antipsychotic medications can potentially affect overall voluntary movements and swallowing movements because of their antagonism of the $\mathrm{D}_{2}$ receptors in patients with PD $[38,39]$. This increased risk of aspiration pneumonia is of particular concern in older patients with PD [40]. Our study revealed that clozapine led to the highest risk of pneumonia in older people with PD. This finding is supported by the fact that clozapine has strong anticholinergic effects [18, 19], in addition to inflammatory effects [27].

The limitations of this study are as follows. First, information on some factors affecting pneumonia are unavailable on the LHID, such as alcohol and tobacco consumption behavior and laboratory measurements. There is also no information about the health status and physical performance, such as self-rated health, activities of daily living (ADL), and instrumental activities of daily living (IADL) disability. Furthermore, the LHID only includes information that is part of the health insurance declaration, and medical information from self-funded medical treatment cannot be obtained. Thus, antipsychotic use may be underestimated. Second, the study only used ICD codes to define the disease without consideration of medical procedure codes; this approach may lead to overdiagnosis. Third, some medications that may potentially increase the risk of incident pneumonia were not enrolled in the study, such as proton pump inhibitors (PPIs). In order to focus on the antipsychotics, the study reduced the medication confounding by adjusting comorbidity disease. Besides, NHI has a strict payment guideline for PPIs that are only paid for severe gastrointestinal diseases. Fourth, all participants in the study were above 65 years old. Since older patients are already at higher risk for pneumonia, future studies can establish a younger PD comparison group to verify the effect of antipsychotics on older patients with PD. In addition, the severity of PD and the disease duration of PD may also affect the study results. This study was a nationwide population-based study. Thus, the study results have the accuracy and representativeness. Besides, its observational research design precluded this study from inferring that antipsychotic use causes pneumonia. Future studies should obtain more information from other databases or through questionnaires to infer causality.

In conclusion, older patients with PD receiving typical antipsychotics or atypical antipsychotics had a higher risk of pneumonia. Among these antipsychotics, clozapine had the highest risk of pneumonia. Clinicians should pay attention to the risk of pneumonia in older patients with PD who receive typical antipsychotics and atypical antipsychotics, especially when prescribing antipsychotics with clozapine, to minimize adverse effects.

\section{Abbreviations}

PD: Parkinson's disease; AGS: American Geriatrics Society; LHID: longitudinal health insurance database; HWDC: Health and Welfare Data Science Center Taiwan; MOHW: Ministry of Health and Welfare Taiwan; NHI: National Health Insurance; ICD-9-CM: International Classification of Diseases, Ninth Revision, Clinical Modification; ICD-10-CM: International Classification of Diseases, Tenth Revision, Clinical Modification; COPD: chronic obstructive pulmonary disease; aOR: adjusted odds ratios; CI: confidence interval; ADL: activities of daily living; IADL: instrumental activities of daily living; PPIs: proton pump inhibitors.

\section{Acknowledgements}

We are grateful to Chung Shan Medical University, Taiwan, China Medical University, Taiwan, Asia University, Taiwan, and the Health Data 
Science Center, China Medical University Hospital, for providing administrative, technical, and funding support that has contributed to the completion of this study. This study is based, in part, on data released by the Health and Welfare Data Science Center, Ministry of Health and Welfare. The interpretation and conclusions contained herein do not represent those of the Ministry of Health and Welfare.

\section{Funding}

This research was funded by the Ministry of Science and Technology Taiwan (MOST 106-2410-H039-007), Chung Shan Medical University Taiwan (CSMU-INT-109-07), and China Medical University Taiwan (CMU109-MF-120).

\section{Ethics Committee Approval}

This study was approved as an ethical review by the Institutional Review Board of China Medical University Hospital, Taiwan (No. CMUH107-REC2004). Owing to the anonymity of the database, the requirement for informed consent was waived.

\section{Author Contributions}

Conceptualization, Kuang-Hua Huang, Wei-Yin Kuo and Chien-Ying Lee; Data curation, Yu-Hsiang Kuan and Yu-Chia Chang; Formal analysis, Yu-Hsiang Kuan, Yu-Chia Chang and Tung-Han Tsai; Methodology, Yu-Hsiang Kuan and Chien-Ying Lee; Validation, Kuang-Hua Huang, Wei-Yin Kuo and Chien-Ying Lee; Writing - original draft, Kuang-Hua Huang, Wei-Yin Kuo and Chien-Ying Lee; Writing review \& editing, Chien-Ying Lee.

\section{Competing Interests}

The authors have declared that no competing interest exists.

\section{References}

1. Fahn S, Sulzer D. Neurodegeneration and neuroprotection in Parkinson disease. NeuroRx. 2004; 1: 139-54.

2. Kwon M, Lee JH. Oro-Pharyngeal Dysphagia in Parkinson's Disease and Related Movement Disorders. J Mov Disord. 2019; 12: 152-60.

3. Almirall J, Rofes L, Serra-Prat M, Icart R, Palomera E, Arreola V, et al. Oropharyngeal dysphagia is a risk factor for community-acquired pneumonia in the elderly. Eur Respir J. 2013; 41: 923-8.

4. Akbar U, Dham B, He Y, Hack N, Wu S, Troche M, et al. Incidence and mortality trends of aspiration pneumonia in Parkinson's disease in the United States, 1979-2010. Parkinsonism Relat Disord. 2015; 21: 1082-6.

5. Kalf JG, de Swart BJ, Bloem BR, Munneke M. Prevalence of oropharyngeal dysphagia in Parkinson's disease: a meta-analysis. Parkinsonism Relat Disord. 2012; 18: 311-5.

6. Mehanna R, Jankovic J. Respiratory problems in neurologic movement disorders. Parkinsonism Relat Disord. 2010; 16: 628-38.

7. Friedman JH. Parkinson disease psychosis: Update. Behav Neurol. 2013; 27: 469-77.

8. Grover S, Somaiya M, Kumar S, Avasthi A. Psychiatric aspects of Parkinson's disease. J Neurosci Rural Pract. 2015; 6: 65-76.

9. Trifiro G, Gambassi G, Sen EF, Caputi AP, Bagnardi V, Brea J, et al. Association of community-acquired pneumonia with antipsychotic drug use in elderly patients: a nested case-control study. Ann Intern Med. 2010; 152: 418-25, W139-40
10. Knol W, van Marum RJ, Jansen PA, Souverein PC, Schobben AF, Egberts AC. Antipsychotic drug use and risk of pneumonia in elderly people. J Am Geriatr Soc. 2008; 56: 661-6.

11. Gau JT, Acharya U, Khan S, Heh V, Mody L, Kao TC. Pharmacotherapy and the risk for community-acquired pneumonia. BMC Geriatr. 2010; 10: 45.

12. Nose M, Recla E, Trifiro G, Barbui C. Antipsychotic drug exposure and risk of pneumonia: a systematic review and meta-analysis of observational studies. Pharmacoepidemiol Drug Saf. 2015; 24: 812-20.

13. Chekani F, Holmes HM, Johnson ML, Chen H, Sherer JT, Aparasu RR. Risk of pneumonia associated with atypical antipsychotic use in nursing home residents with Parkinson's disease. J Psychiatr Res. 2019; 117: 116-21.

14. Pepper PV, Goldstein MK. Postoperative complications in Parkinson's disease. J Am Geriatr Soc. 1999; 47: 967-72.

15. Trifiro G. Antipsychotic drug use and community-acquired pneumonia. Curr Infect Dis Rep. 2011; 13: 262-8.

16. Lee CY, Cheng YD, Cheng WY, Tsai TH, Huang KH. The Prevalence of Anticholinergic Drugs and Correlation with Pneumonia in Elderly Patients: A Population-Based Study in Taiwan. Int J Environ Res Public Health. 2020; 17.

17. Chatterjee S, Carnahan RM, Chen H, Holmes HM, Johnson ML, Aparasu RR. Anticholinergic Medication Use and Risk of Pneumonia in Elderly Adults: A Nested Case-Control Study. J Am Geriatr Soc. 2016; 64: 394-400.

18. Kiesel EK, Hopf YM, Drey M. An anticholinergic burden score for German prescribers: score development. BMC Geriatr. 2018; 18: 239.

19. Nery RT, Reis AMM. Development of a Brazilian anticholinergic activity drug scale. Einstein (Sao Paulo). 2019; 17: eAO4435.

20. Aarsland D, Larsen JP, Cummins JL, Laake K. Prevalence and clinical correlates of psychotic symptoms in Parkinson disease: a community-based study. Arch Neurol. 1999; 56: 595-601.

21. Ravina B, Marder K, Fernandez HH, Friedman JH, McDonald W, Murphy D, et al. Diagnostic criteria for psychosis in Parkinson's disease: report of an NINDS, NIMH work group. Mov Disord. 2007; 22: 1061-8.

22. Copeland LA, Mortensen EM, Zeber JE, Pugh MJ, Restrepo MI, Dalack GW. Pulmonary disease among inpatient decedents: Impact of schizophrenia. Prog Neuropsychopharmacol Biol Psychiatry. 2007; 31: 720-6.

23. Marciano WJ, Sirlin A. Erratum: Radiative corrections to neutrino-induced neutral-current phenomena in the $\mathrm{SU}(2) \mathrm{L} \times \mathrm{U}(1)$ theory. Phys Rev D Part Fields. 1985; 31: 213.

24. Haack M, Hinze-Selch D, Fenzel T, Kraus T, Kuhn M, Schuld A, et al. Plasma levels of cytokines and soluble cytokine receptors in psychiatric patients upon hospital admission: effects of confounding factors and diagnosis. J Psychiatr Res. 1999; 33: 407-18.

25. Kuo CJ, Yang SY, Liao YT, Chen WJ, Lee WC, Shau WY, et al. Second-generation antipsychotic medications and risk of pneumonia in schizophrenia. Schizophr Bull. 2013; 39: 648-57.

26. Raaska K, Raitasuo V, Arstila M, Neuvonen PJ. Bacterial pneumonia can increase serum concentration of clozapine. Eur J Clin Pharmacol. 2002; 58: 321-2.

27. Al-Amin MM, Nasir Uddin MM, Mahmud Reza H. Effects of antipsychotics on the inflammatory response system of patients with schizophrenia in peripheral blood mononuclear cell cultures. Clin Psychopharmacol Neurosci. 2013; 11: 144-51.

28. Haack MJ, Bak ML, Beurskens R, Maes M, Stolk LM, Delespaul PA. Toxic rise of clozapine plasma concentrations in relation to inflammation. Eur Neuropsychopharmacol. 2003; 13: 381-5.

29. Seminog OO, Goldacre MJ. Risk of pneumonia and pneumococcal disease in people with severe mental illness: English record linkage studies. Thorax. 2013; 68: 171-6.

30. Chafetz L, White MC, Collins-Bride G, Nickens J. The poor general health of the severely mentally ill: impact of schizophrenic diagnosis. Community Ment Health J. 2005; 41: 169-84

31. Chou FH, Tsai KY, Chou YM. The incidence and all-cause mortality of pneumonia in patients with schizophrenia: a nine-year follow-up study. J Psychiatr Res. 2013; 47: 460-6.

32. Won JH, Byun SJ, Oh BM, Kim HJ, Park SJ, Seo HG. Pneumonia risk and its associated factors in Parkinson's disease: A National Database Study. J Neurol Sci. 2020; 415: 116949.

33. Jethwa KD, Onalaja OA. Antipsychotics for the management of psychosis in Parkinson's disease: systematic review and meta-analysis. BJPsych Open. 2015; 1: 27-33

34. By the American Geriatrics Society Beers Criteria Update Expert P. American Geriatrics Society 2015 Updated Beers Criteria for Potentially Inappropriate Medication Use in Older Adults. J Am Geriatr Soc. 2015; 63: 2227-46.

35. By the American Geriatrics Society Beers Criteria Update Expert P. American Geriatrics Society 2019 Updated AGS Beers Criteria(R) for Potentially Inappropriate Medication Use in Older Adults. J Am Geriatr Soc. 2019; 67: 674-94.

36. Weintraub D, Chiang C, Kim HM, Wilkinson J, Marras C, Stanislawski B, et al. Association of Antipsychotic Use With Mortality Risk in Patients With Parkinson Disease. JAMA Neurol. 2016; 73: 535-41.

37. Chekani F, Holmes HM, Johnson ML, Chen H, Sherer JT, Aparasu RR. Use of Atypical Antipsychotics in Long-Term Care Residents with Parkinson's Disease and Comorbid Depression. Drug Healthc Patient Saf. 2020; 12: 23-30.

38. Miarons Font $\mathrm{M}$, Rofes Salsench L. Antipsychotic medication and oropharyngeal dysphagia: systematic review. Eur J Gastroenterol Hepatol. 2017; 29: 1332-9. 
39. Bieger D. Neuropharmacologic correlates of deglutition: lessons from fictive swallowing. Dysphagia. 1991; 6: 147-64.

40. Dzahini O, Singh N, Taylor D, Haddad PM. Antipsychotic drug use and pneumonia: Systematic review and meta-analysis. J Psychopharmacol. 2018; 32: 1167-81. 